\title{
Influence of hydrolyzed polyacrylamide hydrogel stiffness on podocyte morphology, phenotype and mechanical properties
}

\author{
Maya Abdallah ${ }^{1,2}$, Marta Martin ${ }^{3}$, Mario Tahchi', Sebastien Balme ${ }^{1^{*}}$, Wissam \\ Faour $^{5}$, Bela Varga ${ }^{3}$, Thierry Cloitre ${ }^{3}$, Orsolya Pall ${ }^{4}$, Frederic J.G. Cuisinier ${ }^{4}$, Csilla \\ Gergely ${ }^{3}$, Maria Bassil2* ${ }^{*}$ Mikhael Bechelany ${ }^{*}$
}

1. Institut Européen des Membranes, ENSCM, CNRS, Université de Montpellier, Montpellier, France.

2. Biomaterials and Intelligent Materials Research Laboratory (LBMI), Lebanese University, Faculty of Sciences II, Physic Department, Lebanon

3. Laboratoire Charles Coulomb, Université de Montpellier, CNRS, Montpellier, France

4. Laboratoire de Bioingénierie et Nanosciences, Université de Montpellier, Montpellier, France

5. Gilbert and Rose-Marie Chagoury School of Medicine, Lebanese American University, Byblos, Lebanon. P.O. Box 36

Corresponding authors: sebastien.balme@umontpellier.fr, maria.bassil@ul.edu.lb, mikhael.bechelany@umontpellier.fr

These authors contributed equally to the work.

Keywords:

Hydrolyzed PAAm Hydrogels, PAAm elasticity, Podocyte, Cells mechanosensitivity 


\section{Graphical abstract:}

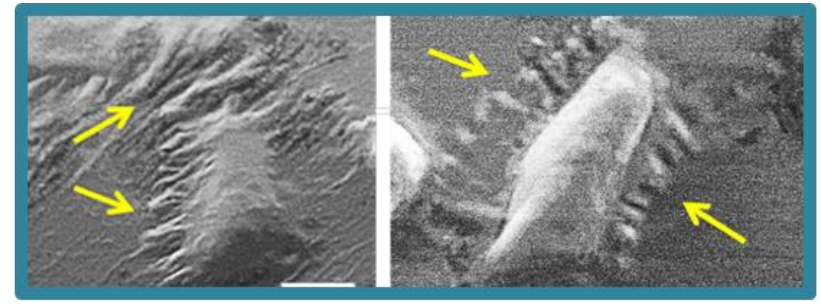

Foot process
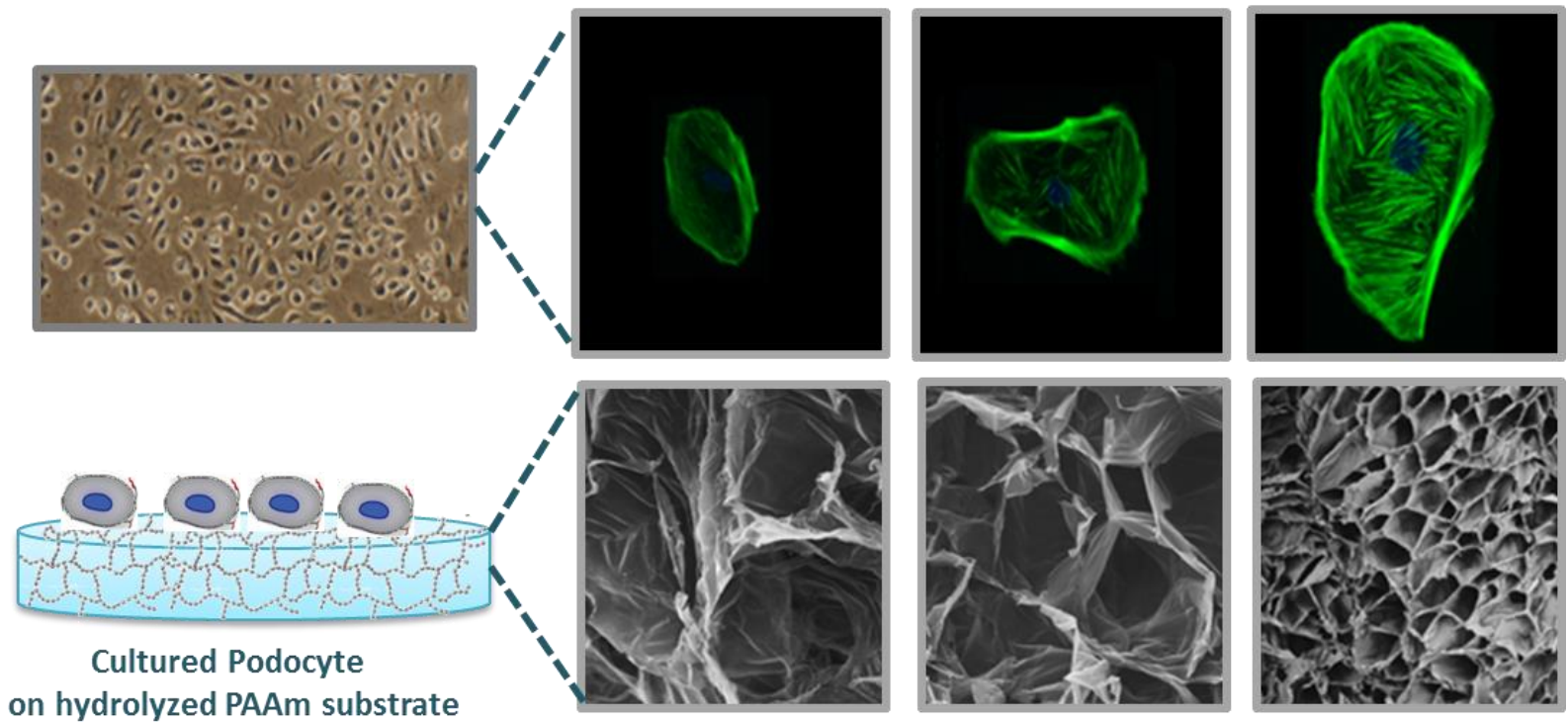

\section{Abstract}

Chronic kidney disease (CKD) is characterized by a gradual decline in renal function that progresses toward end-stage renal disease (ESRD). Podocytes are highly specialized glomerular epithelial cells which form with the glomerular basement membrane (GBM) and capillary endothelium the glomerular filtration barrier (GFB). GBM is an extracellular matrix (ECM) that acts as a mechanical support and provides biophysical signals that control normal podocytes behavior in the process of glomerular filtration. Thus the modulus of elasticity $E$ or stiffness of 
ECM represents an essential characteristic that controls podocyte functions. The biophysical properties of hydrolyzed Polyacrylamide (PAAm) gels resemble to in vivo ECM and thus provide an opportunity to be applied as ECM-like membranes in cellular behavior studies. Therefore, hydrolyzed PAAm hydrogels were investigated for their potential use as new ECM-like constructs to engineer a basement membrane that form with cultured human podocytes a functional glomerular-like filtration barrier. Such ECM-like polyacrylamide hydrogel construct will provide unique opportunities to reveal podocyte cell's biological responses in an in vivo-like setting by controlling the physical properties of the PAAm membranes. In this work, several PAAm hydrogel layers were prepared with different crosslinker concentrations. The macromolecular microstructure and stiffness were evaluated by Scanning Electron Microscopy (SEM) and Atomic Force Microscopy (AFM) techniques respectively. Accordingly, the mechanical properties and the polymeric network porosity can be effectively controlled by modulating the crosslinker concentration as well as the swelling degree. Moreover, modulating gel stiffness significantly influenced podocyte behavior including morphology and actin cytoskeleton reorganization. In conclusion, a strong correlation was observed between podocytes mechanical properties and hydrogels stiffness. This work addressed the complexity of podocytes behavior which will further enhance our knowledge to develop a kidney-on-chip model much needed in kidney function studies on both healthy and diseased states. 


\section{Introduction:}

The development of an in vitro model for cell differentiation and growth has a prior importance for the understanding of fundamental mechanobiology as well as medical applications in the regeneration of damaged organs. To this end, different types of materials have been investigated as a support for cell culture in order to study their influence on cell behavior ${ }^{1}$. These materials permit to develop microfluidic organ-on-a-chip culturing systems that help to establish an appropriate microenvironment for cells and also to mimic a functional unit of human living organ in vitro. Extracellular matrix (ECM) has a major effect on the regulation of cellular behavior such as morphology, proliferation and differentiation. The latter processes can be substantially altered in response to the changes in the chemical composition, stretch, shape and the mechanical properties of the extracellular matrix ${ }^{2}$. Damage of ECM associated with the reduction of tissue integrity and thus loss of resident cells is a phenomenon observed in many chronic illnesses including rheumatoid arthritis as well as chronic kidney disease. Furthermore, recent studies reported that extracellular matrix stiffness is associated with the etiology of several diseases ${ }^{3}$. Thus engineered ECMs were used as culture systems mimicking in-vivo setting in order to understand how ECMs interact and control cellular processes and tissues integrity in both healthy and diseased states ${ }^{4}$.

Synthetic materials such as Polyacrylamide (PAAm) gel ${ }^{5}$ and Polydimethylsiloxane (PDMS) ${ }^{6}$ as well as biomaterials such as collagen or gelatin are the most commonly used in biomedical research field due to their important biomimetic properties. Collagen and gelatin are characterized by their biocompatibility, inherent biodegradability and specific biological functions ${ }^{7}$, since they represent a major component of many connective tissues. Of note, the 
elasto-mechanical properties of connective tissues vary according to the type of collagen as well as the matrix component produced by that tissue. Interestingly, ECM collagen content is remodeled throughout the life span and degraded connective is continuously replaced with newly synthesized collagen. However, these hydrogels are limited by a low stiffness ( $<1 \mathrm{kPa})$ and short-term stability ${ }^{4}$. The PDMS presents an acceptable biocompatibility but does not fully mimic a soft viscoelastic collagen matrix due to its limited scale of stiffness $>5 \mathrm{kPa}^{7}$. Thus it is not considered a suitable GBM alternative to study glomerular filtration barrier function ${ }^{8,9}$. PAAm gels are advantageous because their stiffness can be modulated over a wide range from $0.3 \mathrm{kPa}$ up to $300 \mathrm{kPa}^{10}$. This stiffness variation permits to better understand the cell responses toward the mechanical properties of the support ${ }^{11}$. Although the presence of unreacted acrylamide which is toxic for cells in PAAm network remains an important disadvantage that can be tackled using hydrolyzed polyacrylamide hydrogels.

Hydrolyzed PAAm hydrogels consist of viscoelastic polymer network characterized by a hydrophilic structure ${ }^{12,13}$. They are biocompatible polyelectrolytes capable of absorbing, holding within their interstitial space and releasing on demand large amounts of water or biological fluid. In addition, they can be designed in various 3D structures. They are extensively used in the development of artificial muscles, sensors ${ }^{14,15}$ and drug delivery ${ }^{16}$. During the swelling process, unpolymerized monomers and extractable materials like unreacted Ammonium Persulfate (APS), Tetramethylethylenediamine (TEMED) and their degradation products are washed out from the polymer matrix. Their swelling degree, network microstructure and stiffness ${ }^{17}$ can be easily tuned as desired covering a wide range of physicochemical properties. These characteristics makes them toxin free and permeable to 
small molecules such as oxygen, nutrients, and metabolites that are considered the main advantage allowing cells survival. Thus, hydrolyzed PAAm can be designed to simulate the in vivo environment ${ }^{13}$ and should be regarded as a promising new scaffold material.

The mechanical characteristics of cells microenvironment play a critical role in the regulation of cell behavior. Matrix stiffness is a fundamental physical property that affects cells by modifying their shape, size and cytoskeleton reorganization ${ }^{18}$. Cells responds to cell-ECM stimuli generated by physical constraint of the support by applying counteract forces through cytoskeleton reorganization in order to control cellular functions. Typically, the intensity of these forces generated by the cells depends on ECM stiffness. In fact, each type of cells has a specific mechanical microenvironment which affects its adhesion, migration and proliferation. For instance, efficient proliferation correlates with subtract stiffness and differ from one cell type to another e.g., fibroblasts $(\sim 10 \mathrm{kPa})^{18}$, neurons $(\sim 0.5 \mathrm{kPa})^{19}$, chondrocyte $(\sim 10 \mathrm{kPa})^{20}$ and osteoblast $(-20 \mathrm{kPa})$. Moreover, matrix stiffness can influence the differentiation of mesenchymal stem cells into osteogenic or neurogenic lineages according to matrix stiffness ${ }^{21}$. Most of the cells spread better on a highly stiff substrate, which stimulate the cells to upregulate the expression of stress fibers crucial for keeping healthy cell-ECM interactions ${ }^{22}$. Softer tissues such as liver, breast, and kidney have elasticity on a range between 0.2-4.0 kPa.

The glomerular filtration barrier is composed of podocytes and capillary endothelial cells separated by a glomerular basement membrane (GBM). The latter is considered a soft tissue with an elasticity about $2.5 \mathrm{kPa}^{3}$. A decrease in GBM stiffness below this value is a characteristic of several renal diseases ${ }^{8}$. Podocytes are highly differentiated renal glomerular epithelial cells. They represent an essential component of the glomerular filtration barrier ${ }^{23}$. Podocyte 
structure is characterized by a huge cell body and cytoplasmic foot processes or projections that attach to and cover the entire endothelium. These "foot processes" interdigitate together in a zipper like structure to form molecular filtration units named "slits diaphragm" 24. Podocytes are the functional element for filtering blood in the glomerulus. Their sieving function properties are tightly related to their differentiation and phenotype state, which are both influenced by the mechanical and chemical properties of the matrix ${ }^{7}$. Hu et al. have reported that the effect of gelatin-mTG substrate stiffness has a great impact on nephrin and podocin expression. These proteins are essential to form a functional slit diaphragm and are involved in the mechanosensation of human podocytes ${ }^{25}$.

Hydrolyzed polyacrylamide hydrogels are able to hold cell medium, which together with conditionally immortalized human podocytes give the possibility to develop a 3D model of glomerular filtration barrier. However, the optimal mechanical properties of hydrolyzed polyacrylamide hydrogels that will preserve an intact "slit diaphragm" and foot processes structures remains a key question. Here, we hypothesized that the modulation of the crosslinker to decrease the mesh size of the gel will thus increase its stiffness ${ }^{26}$. When the latter will be within the glomerular stiffness range, we expect to reach such optimal conditions, which is a crucial step towards the development of a kidney-on-chip model.

This work aims at investigating the possibility of using the hydrolyzed form of PAAm hydrogel as a scaffolding material and studying the effect of matrix mechanical and structural properties on podocytes morphology and mechanical properties. Six PAAm Hydrogels layers were prepared by changing the crosslinker concentration. We showed that varying the crosslinker concentration, the swelling degree, the mechanical properties and the polymeric network 
density can be controlled over a wide range as well. The macromolecular microstructure and stiffness of the support were evaluated by Scanning Electron Microscopy (SEM) and Atomic Force Microscopy (AFM) respectively. Then, podocytes were grown on hydrolyzed PAAm support. Their morphology and mechanical properties were evaluated in order to investigate and understand how podocytes sense and respond to the mechanical properties of the substrate.

\section{Materials and Methods:}

\section{Fabrication of Hydrolyzed Polyacrylamide Hydrogels:}

\section{a) Fabrication of Polyacrylamide Gel:}

The solution of Acrylamide (AAm, Sigma Aldrich, A8887, 79-06-1, purity >99\%) and $N, N^{\prime}-$ Methylenbisacrylamide (Bis-acrylamide, Sigma Aldrich, M7279, 110-26-9 purity >99.5\%) were prepared at a concentration of $30 \%$ and $2 \%$ respectively. Gel solutions were prepared by mixing a constant concentration of AAm with various Bis-acrylamide concentrations (Table 1). The free radical polymerization reaction is initiated and catalyzed by using Ammonium Persulfate (APS, Sigma Aldrich, 248614, 7727-54-0, purity >98\%) and $N, \quad N, N^{\prime}, \quad N^{\prime}$ Tetramethylethylenediamine (TEMED, Sigma Aldrich, T7024, 110-18-9) respectively. After mixing, the gel solution was poured into a rectangular rubber mold between 2 glass slides $(6 \mathrm{~cm}$ $\times 2 \mathrm{~cm} \times 0.8 \mathrm{~cm}$ ) and left for $3 \mathrm{hrs}$ at room temperature to achieve polymerization. 


\begin{tabular}{|c|c|c|c|c|}
\hline Samples & $\begin{array}{c}\text { Acrylamide 30 \% } \\
(\mathbf{m l})\end{array}$ & $\begin{array}{c}\text { Bisacrylamide 2 \% } \\
(\boldsymbol{\mu l})\end{array}$ & $\begin{array}{c}\text { APS 25 \% } \\
(\boldsymbol{\mu l})\end{array}$ & TEMED $(\boldsymbol{\mu l})$ \\
\hline M1 & 1 & 0.5 & 1 & 1 \\
\hline M2 & 1 & 1 & 1 & 1 \\
\hline M3 & 1 & 2 & 1 & 1 \\
\hline M4 & 1 & 5 & 1 & 1 \\
\hline M5 & 1 & 10 & 1 & 1 \\
\hline M6 & 1 & 30 & 1 & 1 \\
\hline
\end{tabular}

\section{b) From Gel to Hydrogel:}

\section{- Hydrolysis and Swelling:}

After complete polymerization, PAAm gel layers were gently removed from the mold and were placed overnight in a basic solution of sodium hydroxide (1 M) ( $\mathrm{NaOH}$, Sigma Aldrich, S8045, 1310-73-2, purity > $98 \%$ ) to obtain a hydrolyzed PAAm gel. The hydrolysis reaction induces the conversion of amine moieties $\left(\mathrm{R}^{-\mathrm{NH}_{2}}\right)$ of PAAm network chain into carboxylate (-COO-) moieties which are characterized by their high affinity for water. The gel is then placed in deionized water (MilliQ, conductivity higher than $18 \mathrm{M} \Omega$ ). Upon reaching swelling equilibrium in water, the resulting hydrogels are gently removed and placed in complete cell medium.

\section{- Swelling Measurement:}

After reaching the equilibrium swelling degree in deionized water and cell media, the swelling degree was measured. It is defined as the ratio of the network weight in the swollen state over the dry state. (Equation 1)

$$
S=\frac{W_{S}-W_{d}}{W_{d}}
$$


Where $W_{s}$ and $W_{d}$ are the weight of a fully swollen (in equilibrium with water and cell media) and dehydrated Hydrogels respectively. Measurements were repeated 3 times.

\section{Rheology of PAAm Hydrogel:}

Anton Paar Physica MCR 301 rheometer was used to characterize the mechanical properties of PAAm hydrogels fully swelled in cell media. In this study, a metal plate (Anton Paar PP25, 25mm in diameter) was used to apply an oscillatory force on the surface of the hydrogel to characterize the viscoelastic properties. A frequency of $1 \mathrm{~Hz}$, strain amplitude of $1 \%$ and a fixed force of $0.5 \mathrm{~N}$ were applied. The rheology measurements were recorded at a temperature of $37^{\circ} \mathrm{C}$.

$G^{\prime} G^{\prime}+G^{\prime \prime}(2)$

$\mathrm{G}^{\prime}$ is the storage modulus that represents the elastic property meaning the capacity of membrane material to retain a recoverable elastic energy. $G^{\prime \prime}$ is the loss modulus and determined by the loss of energy due to the viscous forces.

\section{Scanning Electron Microscopy (SEM):}

Hydrogel microstructure was evaluated using SEM technique. The samples were placed in liquid nitrogen $\left(-197^{\circ} \mathrm{C}\right)$ for $4 \mathrm{hrs}$ and subsequently lyophilized. The freeze dried samples were processed in a vacuum system (LABCONCO ${ }^{\circledR}$, FreeZone 4.5 ) at $0.02 \mathrm{mBar}$ and $-54^{\circ} \mathrm{C}$ for overnight. The dried samples were gold sputter-coated to be analyzed by SEM (ZEISS, EVO I HD15). 


\section{Atomic Force Microscopy (AFM):}

The AFM experimental system used for force-spectroscopy measurements was an Asylum MFP3D head coupled to a Molecular Force Probe 3D controller (Asylum Research, Santa Barbara, CA, USA). Triangular silicon nitride cantilevers (MLCT, Veeco) with a nominal spring constant of $10 \mathrm{pN} / \mathrm{nm}$, length of $310 \mu \mathrm{m}$, width of $20 \mathrm{~nm}$, resonance frequency of $7 \mathrm{kHz}$, and half-opening angle of $19^{\circ}$ were used. The cantilever spring constant was determined using the thermal noise method available within the MFP-3D software. Hydrogel samples were glued to a Petridish with double-faced adhesive tape, and covered with $0.5 \mathrm{ml}$ of deionized water. After testing a range of loading forces on the hydrogel surface, the measurements were performed in liquid at room temperature with a maximum loading force of $1 \mathrm{nN}$ corresponding to a maximal indentation depth of $0.3 \mu \mathrm{m}$. Elastic deformation was obtained from the recorded force curves as a function of the loading force applied by the tip. Young's modulus $(E)$ was calculated for each force from the approaching part of the curve, using a modified Hertz model ${ }^{27}$, based on the work of Sneddon ${ }^{28}$ and further developed for different AFM tip shapes ${ }^{29,30}$, as described elsewhere ${ }^{31}$. An approach velocity of $6 \mu \mathrm{m} / \mathrm{s}$ was chosen, indicating a piezo-extension rate of $1 \mathrm{~Hz}$ to minimize hydrodynamic and viscoelastic artifacts ${ }^{32}$.

The Poisson's ratio of the cells was assumed to be 0.5 , as suggested for incompressible materials ${ }^{33}$. Furthermore, to investigate cells' nanomechanical properties on each sample, we repeated the force spectroscopy experiment using the same parameters.

\section{Cell Culture}


Human podocyte cell line was purchased from the Faculty of Medicine, University of Bristol, UK. Immortalized human podocytes were cultured on flasks according to the supplier protocol ${ }^{34}$. They are proliferated at $33^{\circ} \mathrm{C}$ and switched to $37^{\circ} \mathrm{C}$ to be differentiated using a RPMI -1640 Medium (Sigma Aldrich, R8758) supplemented with $1 \%$ insulin-transferrin-Selenium liquid media supplement (Sigma Aldrich, 13146), 10 \% fetal bovine serum (Sigma Aldrich, F7524) and Penicillin Streptomycin solution (Sigma Aldrich, P4333). After proliferation, cells were collected and plated on the hydrolyzed PAAm Hydrogel substrate having various mechanical properties. The PAAm substrates were coated with collagen type I (Sigma Aldrich, C3867, 9007-34-5) using EDC (Sigma Aldrich, E6383, 25952-53-8) and NHS (Sigma Aldrich, 130672, 6066-82-6) to be crosslinked with the carboxylic group.

\section{Immunocytochemical characterization}

Podocyte cells cultured on the surface of PAAm Hydrogels for 7 days were fixed with $2 \%$ of paraformaldehyde (PFA) (Sigma Aldrich, P6148, 30525-89-4) for 15min at room temperature. Then, cell permeablization was performed by using $0.5 \%$ Triton X-100 (Sigma Aldrich, X100, 9002-93-1) for $15 \mathrm{~min}$ into the incubator $\left(37^{\circ} \mathrm{C}\right)$. Afterwards, the non-specific protein binding sites were blocked with $1 \%$ of Bovine Serum Albumin (BSA) (Sigma Aldrich, A2153, 9048-46-8) and $0.5 \%$ of Triton-X in PBS during overnight at $4^{\circ} \mathrm{C}$. Cells were then incubated overnight at $4^{\circ} \mathrm{C}$ with a primary anti-podocin antibody (Sigma Aldrich, P0372). The samples were then rinsed with PBS and incubated with secondary anti-rabbit antibody (Alexa Fluor ${ }^{\circledR} 594$, Cat: ab150080) for $1 \mathrm{hr}$ in dark at room temperature. Nucleus and actin cytoskeleton staining were done with DAPI (Sigma Aldrich, D9542) and Phalloidin staining (Invitrogen, Cat: A12379), which were 
applied for $0.3-1 \mathrm{hr}$ in dark at room temperature. Image acquisition was achieved using Nikon TE2000 microscope.

\section{Multiphoton Microscopy (MPM)}

For a high resolution images, podocyte cells were scanned with a custom-built multiphoton microscope based on a SliceScope upright microscope (MPSS-1000P) equipped with a Multiphoton Scan Head (MP-2000), both from Scientifica LTD. For sample excitation, a SpectraPhysics Tsunami Ti-Sapphire laser operated in pulsed mode was used, wavelength range from 760 to $900 \mathrm{~nm}$, typically $870 \mathrm{~nm}$, repetition rate $80 \mathrm{MHz}$; pulse duration $100 \mathrm{fs}$. The laser beam was focused on the sample via a Nikon CFI75 LWD-16x-W objective (NA 0.8, water immersion). MPM images were created by laser raster scanning the sample. The two photon excited fluorescence signal was epi-collected through the objective.

\section{Cell Proliferation Assay}

MTT (3-(4, 5-dimethylthiazol-2-yl)-2, 5-diphenyltetrazoliumbromide) test was used to evaluate podocyte cells proliferation. After 5 days of culture on PAAm hydrogels, $1 \mathrm{mg} / \mathrm{ml}$ MTT solution (Sigma Aldrich, M5655, 298-93-1) was added to cells and incubated for $3 \mathrm{hrs}$ at $37^{\circ} \mathrm{C} . \mathrm{MTT}$ solution was carefully descanted and replaced by Isopropanol (Sigma Aldrich, 19516, 67-63-0) to dissolve the violet formazan crystals. After $1 \mathrm{hr}$ incubation at room temperature in the dark the luminescent signals provided by MTT assay were detected and cell proliferation was determined by normalizing each luminescent signal from cells cultured on gels over cells cultured without gel (control). 


\section{Statistical Analysis}

Data are presented as mean \pm SD. Statistical significance was determined using one-way ANOVA with post hoc Tukey's Honest Significant Difference test for multiple comparisons. P values of less than 0.05 were considered significant.

\section{Results and Discussion}

Correlation between hydrolyzed PAAm Structural and Mechanical Properties and Podocytes Mechanobiology:

PAAm substrates elasticity:

Hydrolyzed PAAm layers having increasing Bis-acrylamide crosslinker concentrations $(0.5,1,2$, 5, 10 and $30 \mu l)$ were prepared and their mechanical properties were evaluated. 
(A)

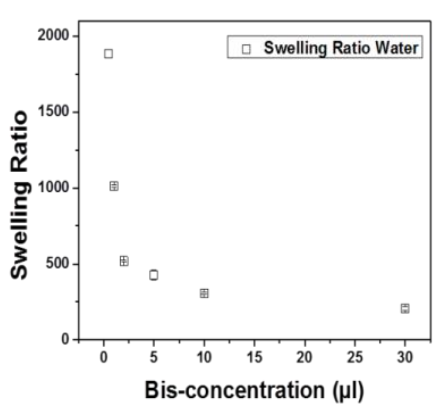

(B)

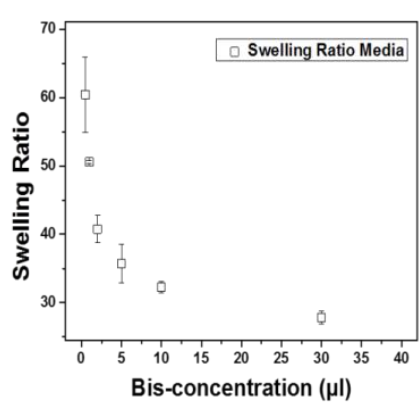

M4

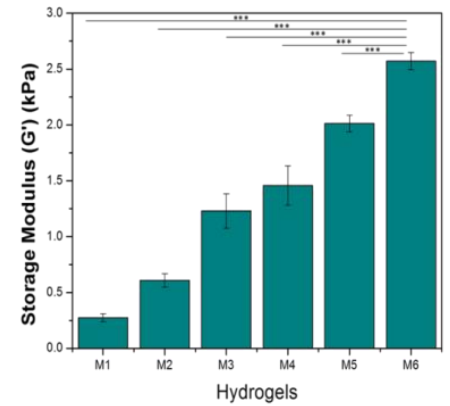

M6
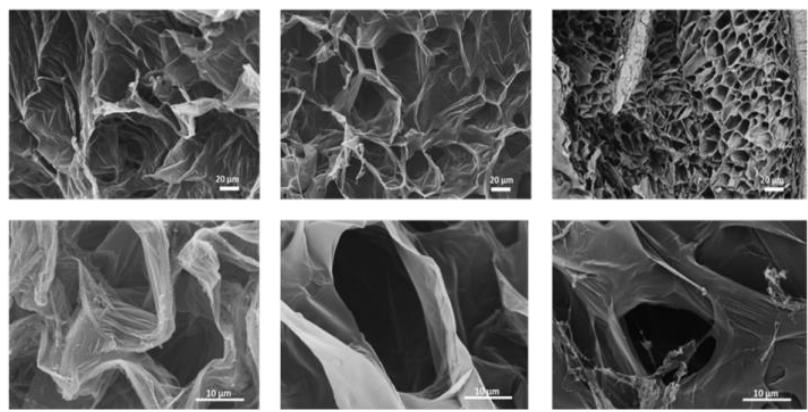

Figure 1: (A) Representative curve of swelling degree as a function of Bis-acrylamide concentration $(0.5,1,2,5,10$ and $30 \mu \mathrm{l})$. (B) Scanning Electron Microscopy images of dehydrated PAAm hydrogels show the polymeric network density as a function of crosslinker concentration: M1 $(0.5 \mu \mathrm{l}), \mathrm{M} 4(5 \mu \mathrm{l}), \mathrm{M} 6(30 \mu \mathrm{l})$. The membrane containing the highest concentration of Bis-acrylamide crosslinker presents a more compacted network than a membrane having a low concentration of crosslinker. (Error Bar = Standard Deviation). (C) The storage modulus of PAAm membranes swelled in cell media was determined as a function of Bis-acrylamide crosslinker concentrations. (Error Bar = Standard Deviation)

The swelling is characterized by the capacity of hydrogels to absorb aqueous medium and to maintain it for a long period. The hydrogel swelling is induced by osmotic forces generated by the hydrophilic $\mathrm{COO}^{-}$and $\mathrm{Na}^{+}$ions entrapped inside the hydrogel matrix, while the 3D structure is maintained due to the mechanical forces of the crosslinked network. The swelling degree was measured for PAAm Hydrogels fully swollen in deionized water and in cell medium. An 
exponential decrease of swelling ratio from 1914 to 193 and from 54 to 26 with increasing crosslinker concentration from $0.5 \mu \mathrm{l}$ to $30 \mu \mathrm{l}$ was determined in water and cell medium respectively (Fig. 1A). Thus an increase in Bis-acrylamide crosslinker concentration contributes to a significant decrease in the swelling ratio. The density of PAAm network evaluated by Scanning Electron Microscopy (SEM) shows that the highest concentration of Bis-acrylamide provides a highly connected structure (Fig. 1B). Consequently, the polymer network becomes more compact and thus less prone to absorb water. This indicates that the crosslinking density of polymer chain has an influence on the swelling capacity. Previous studies have investigated the effect of crosslinker concentration of polyacrylamide hydrogel on the swelling mechanism and have exhibited that an increase of crosslinking density contributes to low gels capacity to be swelled due to highly crosslinked polymer network ${ }^{35}$.

For a macroscopic characterization of mechanical properties, the viscoelasticity of hydrogels was determined by applying an oscillatory force on the total surface of PAAm gel fully swelled in cell medium. The elastic and viscous properties are represented by the storage modulus and the loss modulus respectively. The storage modulus is defined by the membrane capacity to hold a recoverable energy and the loss modulus is characterized by the dissipated energy. For a low concentration of crosslinker $(0.5 \mu \mathrm{l})$, the storage modulus was about $274 \mathrm{~Pa}$ and increased until $2.5 \mathrm{kPa}$ for a high crosslinker concentration (30 $\mu$ l) (Fig. 1C). Many studies have been worked on the characterization of polyacrylamide gels mechanical properties. Grattoni et al. have already analyzed polyacrylamide hydrogels having different polymer and crosslinker concentrations. They have shown that the storage moduli of the gel increased significantly with the polymer and crosslinker concentrations ${ }^{36}$. In our work, the rheology measurements show as 
expected, an increase of storage modulus with the concentration of crosslinker agent. This indicates that a densely connected network requires more energy to be deformed.

(A)
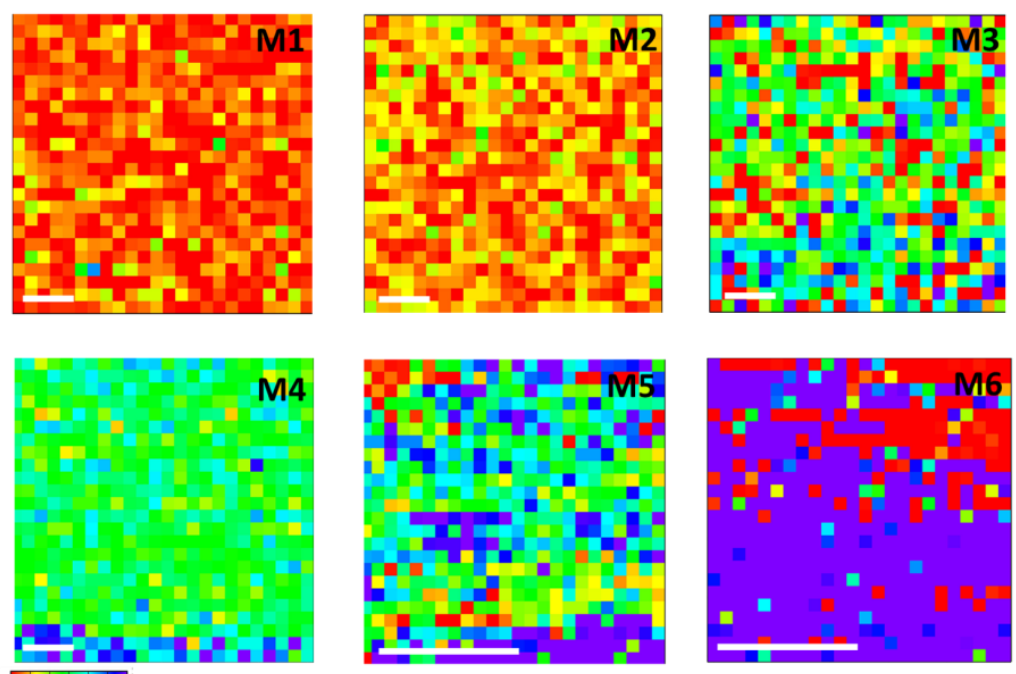

(B)

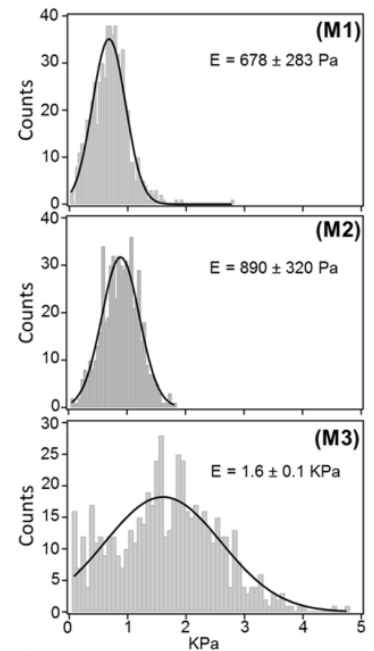

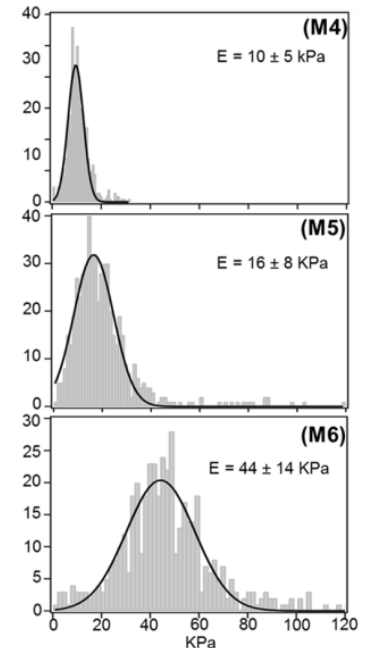

(C)

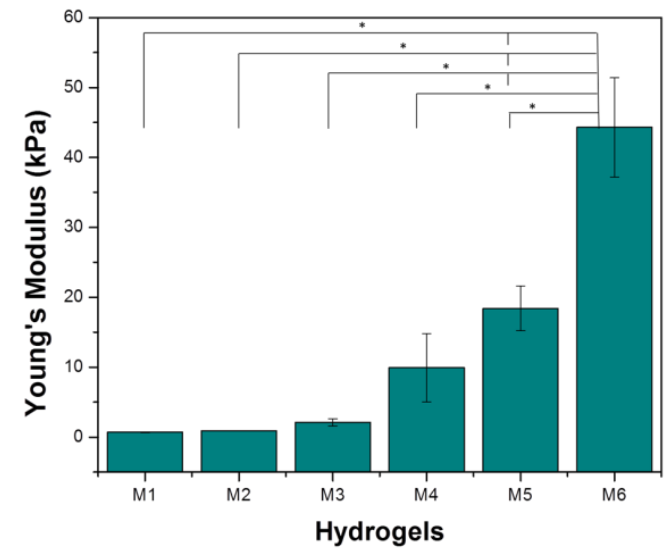

Figure 2: (A) Polyacrylamide hydrogels Young's modulus maps measured by AFM. M1, M2, M3, M4, M5 and M6 samples correspond to bis-acrylamide crosslinker concentrations of 0.5 , 1, 2, 5, 10 and $30 \mu \mathrm{l}$ respectively. Scale bar $5 \mu \mathrm{m}$. Dark red color depicts softer portions, while blue and purple colors show stiffer regions. Color scale bar for (M1), (M2) and (M3) goes from 0 to $3 \mathrm{kPa}$. Color scale bar for (M4), (M5) and (M6) goes from 0 to $30 \mathrm{kPa}$. (B) Elasticity distribution of PAAm hydrogels for M1, M2, M3, M4, M5 and M6 samples (one trial). Young's moduli were best fitted with Gaussian distributions. (C) Evolution of the Young's modulus (E) as a function of bis-acrylamide crosslinker concentrations. The Young's modulus increases 
with increasing concentration of the crosslinker. Bars represent the obtained average of the Gaussian peaks fitted on the elasticity value distribution of individual force maps. Error bars are standard deviation of the mean. * denotes statistical significance of $P<0.05$

To investigate the local mechanical properties, PAAm hydrogel's elasticity was evaluated by force-spectroscopy measurements. The stiffness of PAAm hydrogels defined by the Young's modulus indicates the strength of the support. For all samples, force-volume images were constructed from force curves collected at each point in two-dimensional scan. Typical images are gathered in Fig. 2A for PAAm gels with different crosslinker concentrations. For the softest substrates (M1, M2 and M3), the local distribution of Young's modulus is homogenous. Conversely, for the stiffest supports (M5 and M6), the inhomogeneity of the surface is obvious. The maps indicate a presence of large areas having a very low elasticity comparing to the overall elasticity. For example, the stiffness map of M6 substrate shows areas with a stiffness ranging from 0 to $5 \mathrm{kPa}$, whereas the overall elastic modulus of this substrate is about $44 \mathrm{kPa}$.

The histograms of Fig. 2B are plotted from the local measurement of Young's modulus maps. The average Young's modulus values of $M 1, M 2, M 3, M 4, M 5$ and M6 deduced from the Gaussian fit are $0.67 \pm 0.05 \mathrm{kPa}, 0.90 \pm 0.06 \mathrm{kPa}, 2.1 \pm 0.5 \mathrm{kPa}, 9.9 \pm 4.9 \mathrm{kPa}, 18.4 \pm 3.2 \mathrm{kPa}$ and 44.3 $\pm 7.1 \mathrm{kPa}$ respectively (Fig. $2 \mathrm{C}$ ). Our data show an exponential increase in Young's modulus with increasing crosslinker concentration. These results are comparative with previous studies that have shown an increase in Young's modulus with increasing the crosslinker concentration $^{22}$.

Moreover, there is a correlation with the rheology measurements where an increase of storage modulus was observed. This can be explained by the density degree of the polymer network 
characterized by SEM. At this stage, the swelling properties, the polymer network density and the mechanical properties were perfectly correlated. Typically, the increase of crosslinker concentration contributes to a highly crosslinked polymer making the gel stiff and less prone to the swelling. At the same time, these PAAm hydrogels have a high Young's and loss modulus explained by their highly connected structure with small mesh size that contained less water. Finally, the network with high connectivity is not homogeneous since they exhibit large zones having significantly lower Young's modulus. Contrariwise, the softest gel presents a more homogeneous network. Therefore, hydrogels present an inhomogeneous distribution of crosslinking density. The inhomogeneity degree increases with crosslinker concentration due to the increase of network imperfections establishing less and more crosslinked regions ${ }^{37}$.

Our study seeks to determine a substrate having elasticity close to in vivo state. Since the glomerular basement membrane of the kidney has an elasticity of $2.5 \mathrm{kPa}^{3}$, the substrates $\mathrm{M} 2$, $\mathrm{M} 3$, and M4 (with elasticity of $0.9 \mathrm{kPa}, 2.1 \mathrm{kPa}$ and $9.9 \mathrm{kPa}$ respectively) are in the good range of elasticity of kidney extracellular membrane. Consequently, we have investigated the effect of all the hydrogels on the cellular behavior.

\section{Influence of support mechanical properties on podocyte cells:}

\section{Morphology, Elasticity, Cytoskeleton reorganization and Podocin expression}


(A)

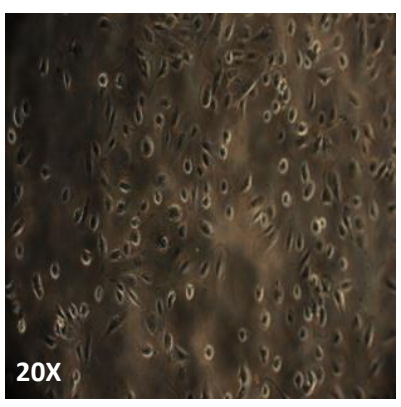

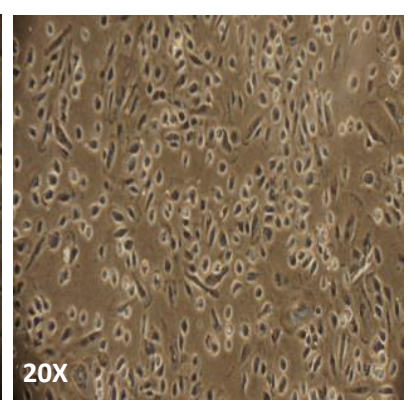

(B)

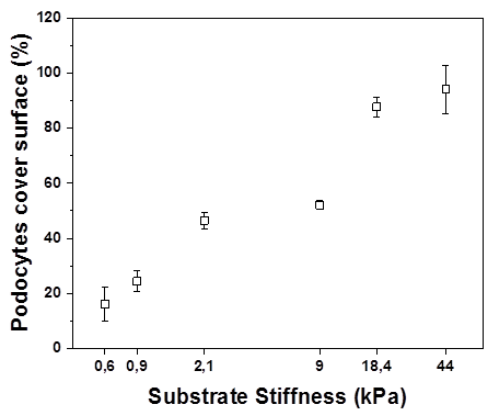

Figure 3: (A) Human Podocyte Cells Line cultured on PAAm hydrogels substrates having different properties (M3 and M5) at a magnification of 20X. (B) Podocyte proliferation: \% of podocytes cover surface as a function of substrate's elastic modulus. (Reference: 100\% for podocytes seeded on the plate)

Podocytes were cultured on PAAm gels covering an elasticity range from $0.6 \mathrm{kPa}$ to $44 \mathrm{kPa}$ in order to evaluate the influence of support stiffness on their morphology and mechanical properties. MTT assay was performed to confirm cell growth and survival on the PAAm hydrogels. Figure 3 shows that podocytes were able to proliferate on all hydrogels. This means that the hydrogel is non-toxic and thus all unreacted acrylamide was removed during swelling process. However, we can notice that the proliferation rate of podocyte cells increases with the gel stiffness. 
(A)

(B)
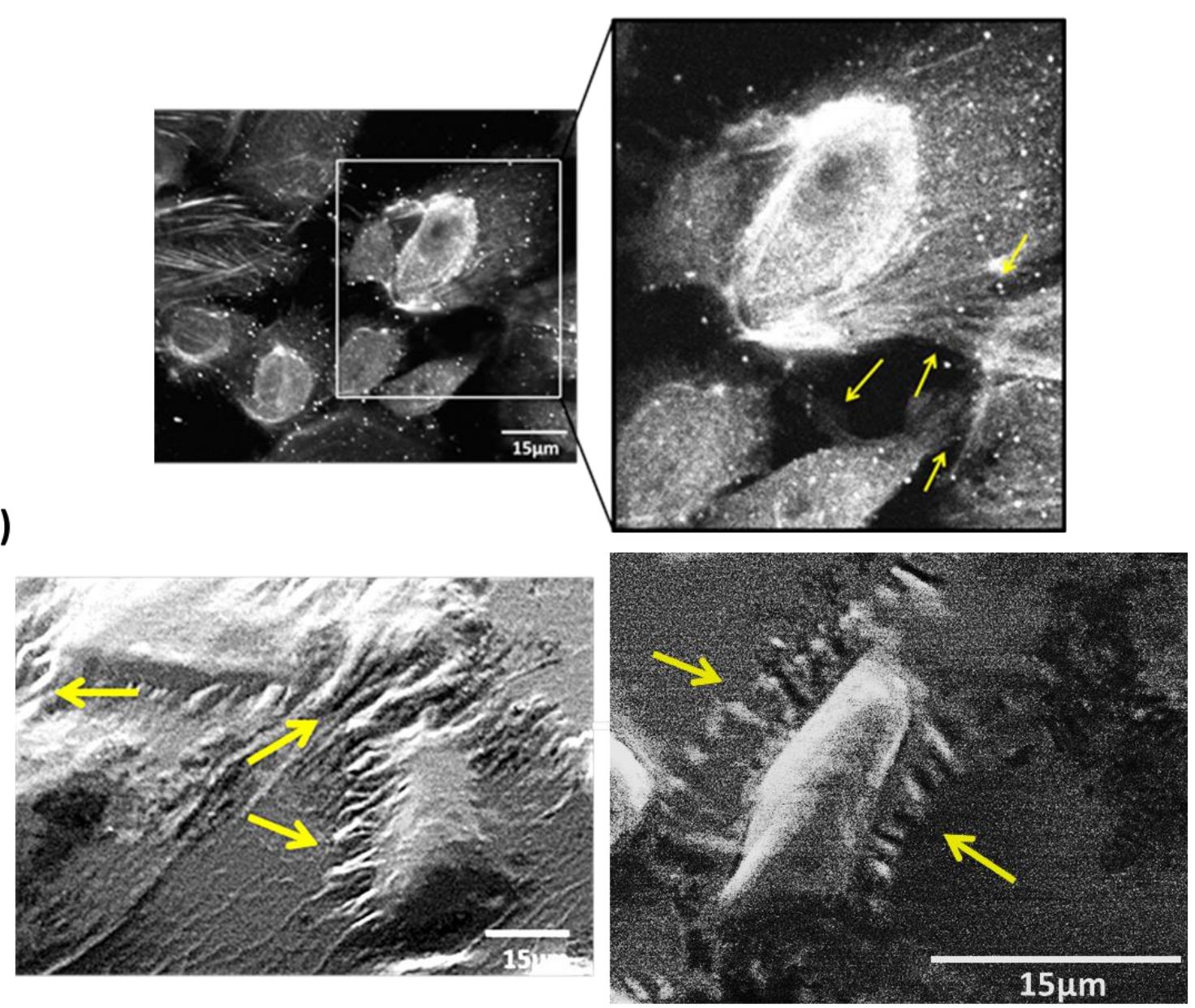

Figure 4: Immortalized podocytes showed an arborized morphology and interdigitating foot processes with adjacent cells (yellow arrow). These images were taken using multiphoton microscopy (A) and environmental SEM (B) for M3 substrate.

Furthermore, multiphoton microscopy and Environmental SEM (Fig. 4) showed that the morphology of conditionally immortalized human podocytes seeded on PAAm hydrogels was comparable to the previously described organoid-derived podocytes in culture ${ }^{38}$ as well as the fully differentiated immortalized human podocyte previously reported ${ }^{34}$. Typically, the 
microscopy images showed the presence of arborized projections that can be described as "foot processes". The latter enable possible cell-cell connections (yellow arrows in Fig. 4) mimicking the "slit diaphragm" responsible for the filtration function of the podocyte layer. The two photon excited fluorescence images measured by MPM reveal the cytoskeletal organization of the podocytes and the interdigitating foot processes with adjacent cells (yellow arrows in Fig. 4). 
(A)

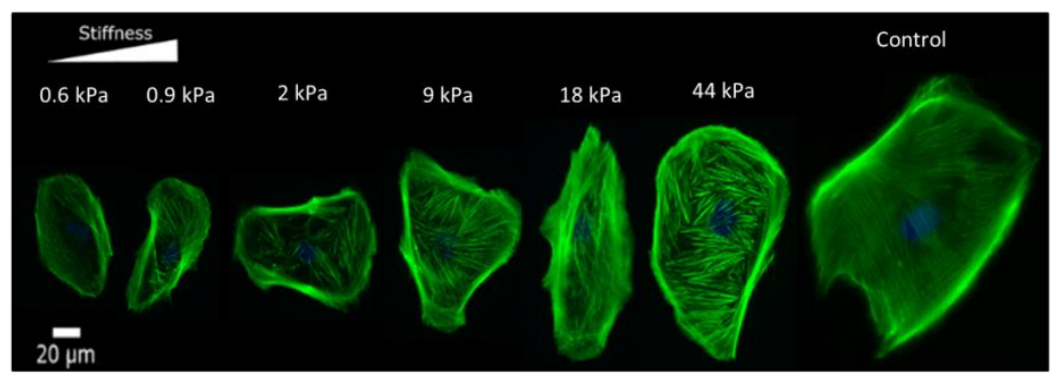

(B)

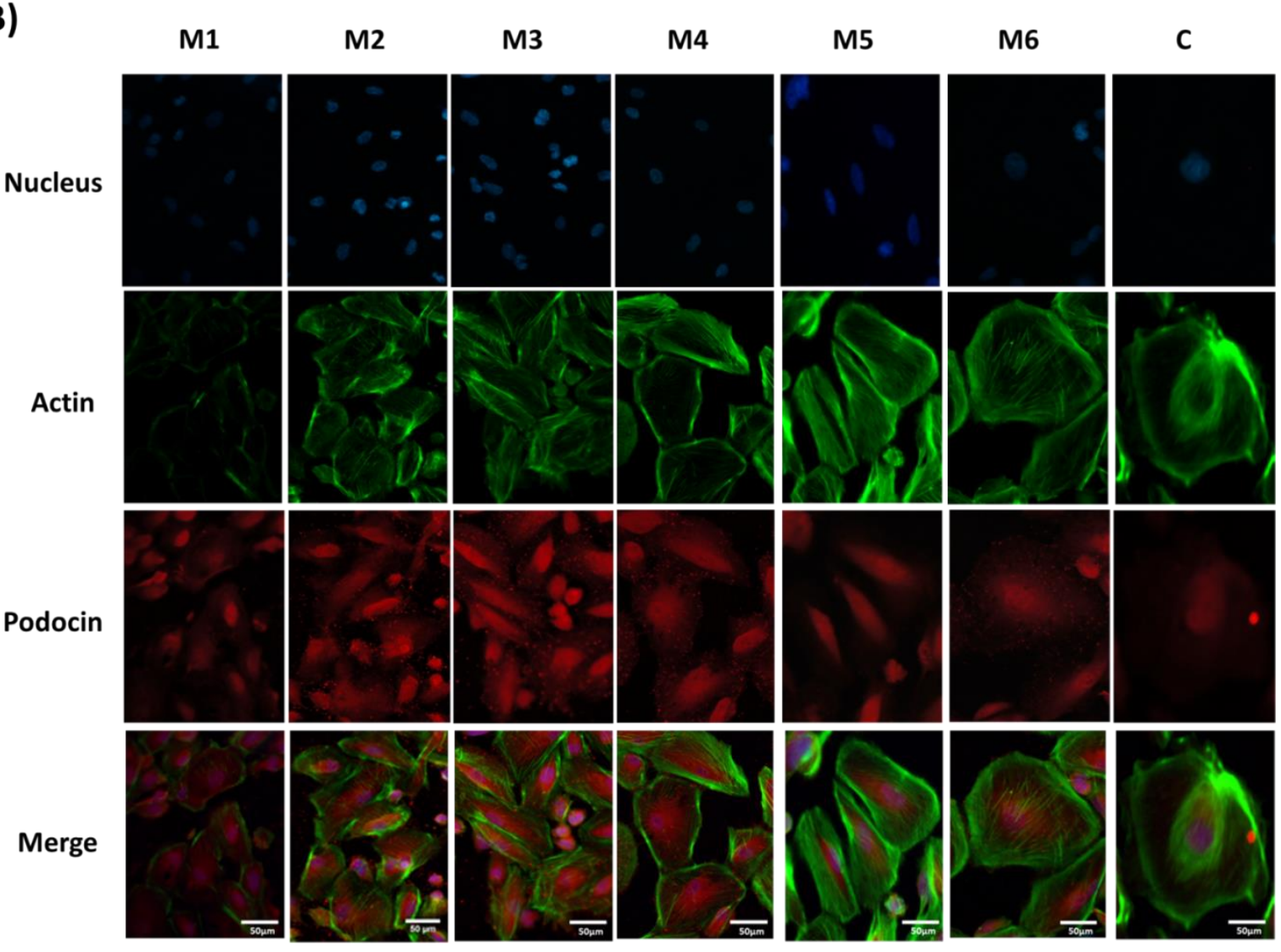

Figure 5: (A) (B) Representative immunofluorescence images of podocytes cultured on PAAm substrates with an elasticity ranging from $0.6 \mathrm{kPa}$ to $44 \mathrm{kPa}$. The cells were detected by staining the nucleus (DAPI-blue), the actin cytoskeleton (Phalloidin-green) and Podocin protein (anti-Podocin-red). Scale bar $50 \mu \mathrm{m}$. Podocyte cells sense and respond to substrates stiffness. Cell morphology was found to be elastic modulus dependent. Podocin expression is 
highly upregulated on PAAm membranes M2, M3 and M4 having elasticity of $0.9 \mathrm{kPa}, 2 \mathrm{kPa}$ and $9.9 \mathrm{kPa}$ respectively, but decreased on the stiffest membranes.

The mechanical properties of support have a clear effect on the morphology and the cytoskeletal reorganization of podocyte cells, which was characterized by visualizing the actin network using Phalloidin staining (Fig. 5). On the softest gel (0.67 kPa), the cells showed a small spread area. They become more and more spread as the gel Young's modulus increases.

The cells mechanosensitivity against ECM is usually explained by the activity of adhesion molecules and non-muscle myosin that produce support stiffness dependent - forces ${ }^{39}$. CellECM attachment involves numerous cytoplasmic linkage proteins including integrins that connect ECM to actin filaments. Integrin play thus a crucial role in cytoskeleton reorganization and cell cycle regulation. For a matrix having high stiffness, the cells respond to mechanical strain by increasing integrin aggregation and therefore an increase in the formation of actin stress fibers. In order to characterize that, actin cytoskeleton reorganization was analyzed in cells cultured on gels with different elasticity. Importantly, actin network formed a diffuse cytoskeleton with a random arrangement for soft substrates (0.67 kPa to $2.1 \mathrm{kPa})$. In contrast, cells cultured on stiff gels reacted in response to mechanical stress by forming a dense actin cytoskeleton (Fig. 5A). The latter scenario is opposite to a random actin cytoskeleton reorganization previously reported on soft substrates ${ }^{18,22,39}$. Finally, the actin cytoskeleton is also oriented and more dense allowing to apply contractile forces to that counterbalance substrate stiffness ${ }^{3}$. 
Healthy podocytes integrity and phenotype are determined by their ability to express specific markers such as podocin, nephrin and CD2AP, which are responsible in the formation of the slit diaphragm ${ }^{40}$. Apart from this, podocytes express Wilms' tumor suppressor (WT-1) which is a nuclear protein involved in the expression of major podocyte markers ${ }^{41,42}$. The expression of podocin was evaluated by immunostaining method (Fig. 5B). As expected, podocin protein is expressed in podocytes. Importantly, the cells podocin level was found to be higher on substrates having an elasticity range of $0.9 \mathrm{kPa}$ to $9.9 \mathrm{kPa}$ than on the harder substrates. This result is consistent with the previously reported data by Hu et al. who observed the same effect on podocytes phenotype but with gelatin substrates of a Young's modulus within the range of 2-5 $\mathrm{kPa}^{7}$. This range seems to be optimal for podocin expression and, thus, it is considered compatible with the stiffness of the glomerular basement membrane. Briefly, in this range of gel stiffness, podocytes upregulate the expression of podocin and downregulate the formation of actin filaments. Conversely, the stiffest gels upregulated actin stress fibers formation and downregulated podocin expression. These results showed strong correlation between the mechanical properties of a substrate and podocyte cell morphology, actin cytoskeleton reorganization, as well as the expression of podocin. Moreover, Hydrolyzed PAAm hydrogels with an elasticity ranging from $0.9 \mathrm{kPa}$ to $9.9 \mathrm{kPa}$ seems to be optimal and it is consistent with the physiological stiffness of kidney glomerular basement membrane which is $2.5 \mathrm{kPa}$. 
(A)
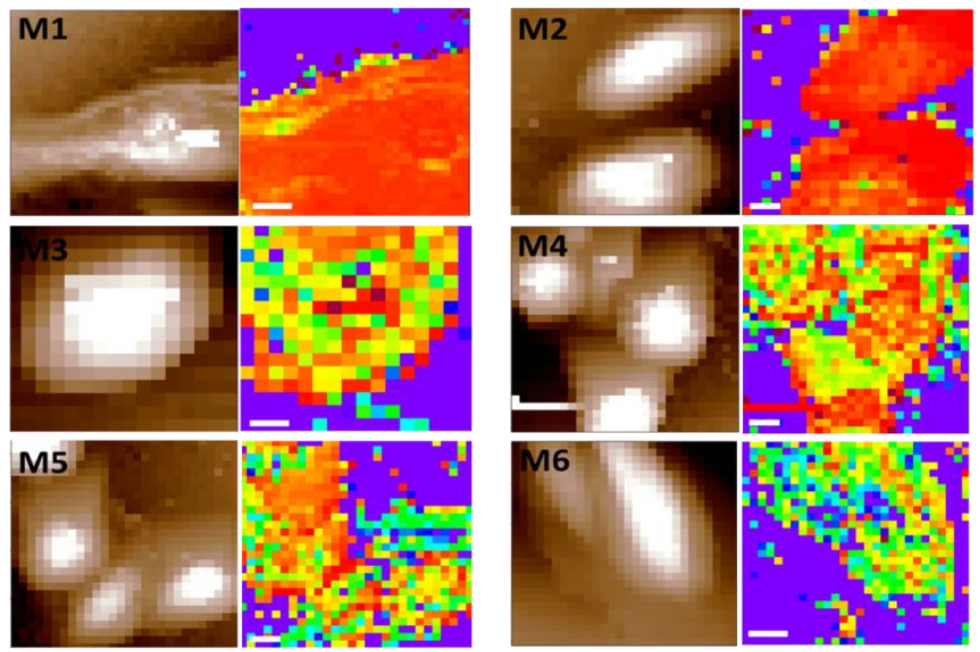

(B)
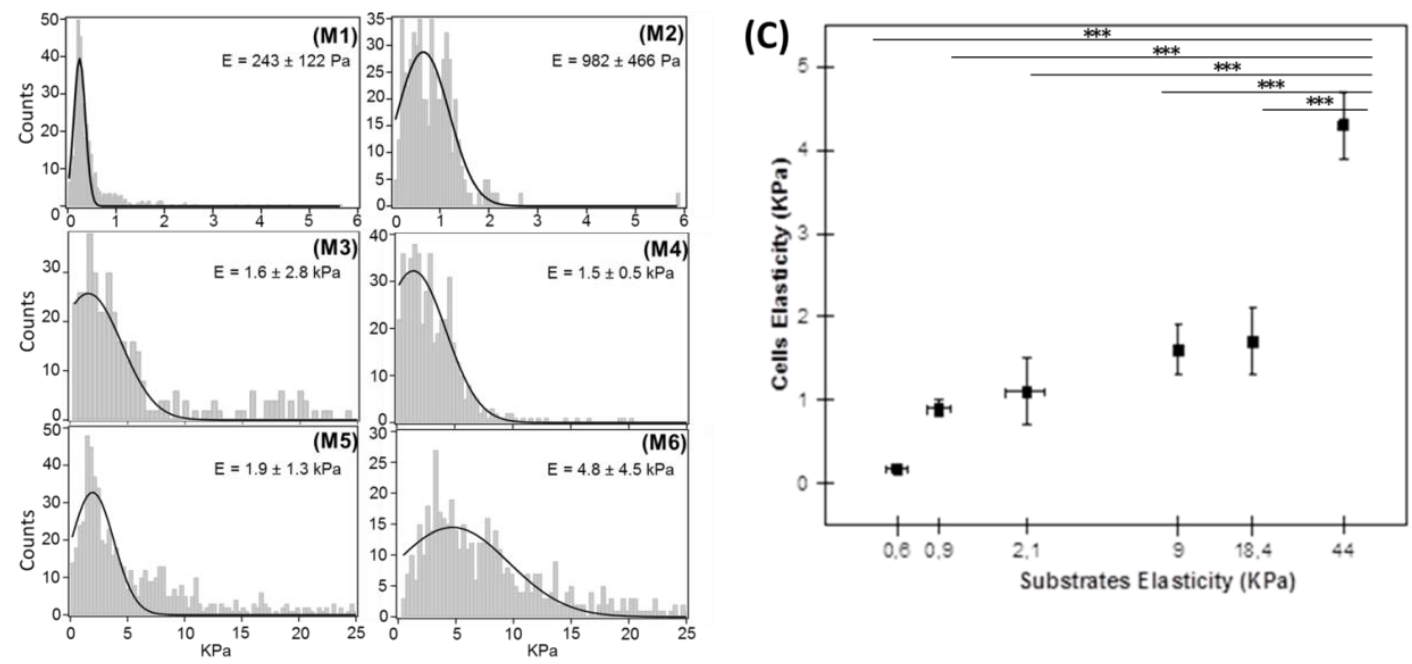

Figure 6: Elasticity of podocytes cultured on PAAm membranes. M1, M2, M3, M4, M5 and M6 represent the height of the contact point and to the Young's modulus map. Dark brown color represents deeper regions, while white depicts the higher portions of the cells. Black scale bar $10 \mu \mathrm{m}$. Color bar goes from $12 \mu \mathrm{m}$ to 0 . Dark red color represents the softer portions, while blue and purple colors show the harder regions. Color bar goes from 0 to $16 \mathrm{kPa}$ (B) AFM elasticity distribution indicating podocyte elasticity for cells cultured on M1, M2, M3, M4, M5 and M6 samples (one trial). Young's moduli were best fitted using Gaussian distributions. (C) Evolution of the cells' Young's modulus (E) as a function of hydrogel stiffness. The Young's 
Modulus increases significantly for podocytes cultured on M6 sample. Bars correspond to the obtained average of the Gaussian peaks fitted on the elasticity value distributions of individual force maps. Error bars are standard deviation of the mean.

Besides podocytes morphology, their mechanical properties can be affected by the substrate stiffness, since the latter play an important role in the formation of actin filaments. Here, AFM was used to provide quantitative measurements on podocytes mechanical properties as a function of PAAm membranes stiffness. Typical force-volume images were gathered in Fig. 6. Panels (M1, M2, M3, M4, M5 and M6) represent the height of the contact point maps recorded during the force-volume measurement and Young's modulus maps for podocytes cultured on hydrolyzed PAAm hydrogels. The Young's modulus maps of podocytes cultured on the softest hydrogels (M1 and M2) showed a rather homogeneous stiffness distribution in contrast with the others. The distribution histograms of Young's modulus values for the different cases are presented in Fig. 6B.

The average Young's modulus values represent the evolution of the increase with hydrogel stiffness. Podocytes cultured on M1, M2, M3, M4, M5 and M6 substrate showed average Young's modulus of $0.18 \pm 0.06 \mathrm{kPa}, 0.9 \pm 0.1 \mathrm{kPa}, 1.1 \pm 0.4 \mathrm{kPa}, 1.6 \pm 0.3 \mathrm{kPa}, 1.7 \pm 0.4 \mathrm{kPa}$ and $4.3 \pm 0.4 \mathrm{kPa}$ respectively. Of note, substrates with Young's moduli ranging from $2 \mathrm{kPa}$ to $18 \mathrm{kPa}$ (M3,M4,M5) does not caused significant variations in the cultured cells elasticity. In contrast, the stiffest hydrogel produced an increase in podocyte stiffness. These results are consistent with the distribution of actin filament shown in Figure 5, which corroborate the data that the actin cytoskeleton network have an essential role in determining mechanical stiffness of the 
cell ${ }^{43}$. Indeed for the substrates M1 and M2 the low values of the Young's modulus can be assigned to the diffusive and random distribution of actin. For substrates M3, M4 and M5, the actin filaments were oriented and their sizes were moderately similar. For the stiffest substrate M6, the actin filaments bundles were clearly larger to counterbalance the mechanical constraints exerted over the podocyte. The size increase of actin filaments can explain the significantly higher Young's modulus of the podocytes.

\section{Conclusion}

In this work, the influence of PAAm hydrogel properties on podocytes was investigated. The Young's modulus of PAAm hydrogels was modulated from $0.06 \mathrm{kPa}$ until $44 \mathrm{kPa}$ with the crosslinker concentration. The latter made the gel network more and more connected and thus less prone to swelling. The PAAm gel is perfectly biocompatible for the podocytes in culture and did not alter their proliferation and viability. However, the substrates strongly influenced podocytes morphology and their mechanical properties. For the softest substrate having a Young's modulus of $0.6 \mathrm{kPa}$, the cells were less spread and were characterized by a low level of podocin expression and a reduction in actin stress fiber formation. For the stiffest substrates $\mathrm{E}$ $=18 \mathrm{kPa}$ and $44 \mathrm{kPa}$, podocyte cells were more spread. The mechanical stress of substrate induced the formation of actin fibers consequent to a downregulation of podocin expression. Podocytes cultured on hydrogel with stiffness ranging between $0.9 \mathrm{kPa}$ and $9.9 \mathrm{kPa}$, which is closer to the elasticity of the glomerular basement membrane provide the best conditions where normal regulation of podocytes markers e.g., podocin, occurs. According to the podocyte mechanical properties, regulation of actin stress fibers formation is optimal for substrates with 
stiffness ranging between $2.1 \mathrm{kPa}$ and $9.9 \mathrm{kPa}$. In addition, the presence of "foot processes" along all the above mentioned conditions makes PAAm hydrogel a worthy design to reproduce glomerular-like filtration process. In conclusion, our results present a new tool that will further contribute to better understand the behavior of podocytes during both healthy and diseased states, and far beyond to develop a microfluidic kidney-on-chip device.

\section{Acknowledgement}

The authors would like to thank the financial support from CEFIPRA (Project 5608-1), the CNRS (Project "Osez I'Interdisciplinarité") and the MUSE project (3DTriatCancer). This project has been funded as well by the National Council for Scientific Research in Lebanon and the Lebanese University.

\section{References:}

1. Chapekar, M. S. Tissue engineering: challenges and opportunities. J. Biomed. Mater. Res. 53, 617-620 (2000).

2. Korolj, A. et al. Curvature facilitates podocyte culture in a biomimetic platform. Lab. Chip 18, 3112-3128 (2018).

3. Janmey, P. A. \& Miller, R. T. Mechanisms of mechanical signaling in development and disease. J Cell Sci 124, 9-18 (2011).

4. Caliari, S. R. \& Burdick, J. A. A practical guide to hydrogels for cell culture. Nat. Methods 13, 405-414 (2016). 
5. Tse, J. R. \& Engler, A. J. Preparation of Hydrogel Substrates with Tunable Mechanical Properties. Curr. Protoc. Cell Biol. 47, 10.16.1-10.16.16 (2010).

6. Palchesko, R. N., Zhang, L., Sun, Y. \& Feinberg, A. W. Development of Polydimethylsiloxane Substrates with Tunable Elastic Modulus to Study Cell Mechanobiology in Muscle and Nerve. PLOS ONE 7, e51499 (2012).

7. $\mathrm{Hu}, \mathrm{M}$. et al. A biomimetic gelatin-based platform elicits a pro-differentiation effect on podocytes through mechanotransduction. Sci. Rep. 7, 43934 (2017).

8. Wyss, H. M. et al. Biophysical properties of normal and diseased renal glomeruli. Am. J. Physiol. - Cell Physiol. 300, C397-C405 (2011).

9. Derieppe, M. et al. Detection of intrarenal microstructural changes with supersonic shear wave elastography in rats. Eur. Radiol. 22, 243-250 (2012).

10. Syed, S., Karadaghy, A. \& Zustiak, S. Simple Polyacrylamide-based Multiwell Stiffness Assay for the Study of Stiffness-dependent Cell Responses. J. Vis. Exp. JoVE. 97, 52643 (2015).

11. Wells, R. G. The role of matrix stiffness in regulating cell behavior. Hepatology 47, 13941400 (2008).

12. Chai, Q., Jiao, Y. \& Yu, X. Hydrogels for Biomedical Applications: Their Characteristics and the Mechanisms behind Them. Gels 3, 6 (2017).

13. Caló, E. \& Khutoryanskiy, V. V. Biomedical applications of hydrogels: A review of patents and commercial products. Eur. Polym. J. 65, 252-267 (2015).

14. Bassil, M., Davenas, J. \& EL Tahchi, M. Electrochemical properties and actuation mechanisms of polyacrylamide hydrogel for artificial muscle application. Sens. Actuators B Chem. 134, 496-501 (2008). 
15. Bassil, M., Ibrahim, M. \& Tahchi, M. E. Artificial muscular microfibers: hydrogel with high speed tunable electroactivity. Soft Matter 7, 4833-4838 (2011).

16. Yahia, Lh. et al. History and Applications of Hydrogels. J. Biomed. Sci. 4, 2 (2015).

17. Bassil, M., El Haj Moussa, G. \& El Tahchi, M. Templating polyacrylamide hydrogel for interconnected microstructure and improved performance. J. Appl. Polym. Sci. 135, 46205 (2018).

18. Yeung, T. et al. Effects of substrate stiffness on cell morphology, cytoskeletal structure, and adhesion. Cell Motil. 60, 24-34 (2005).

19. Flanagan, L. A., Ju, Y.-E., Marg, B., Osterfield, M. \& Janmey, P. A. Neurite branching on deformable substrates. Neuroreport 13, 2411-2415 (2002).

20. Subramanian, A. \& Lin, H.-Y. Crosslinked chitosan: Its physical properties and the effects of matrix stiffness on chondrocyte cell morphology and proliferation. J. Biomed. Mater. Res. A 75A, 742-753 (2005).

21. Engler, A. J., Sen, S., Sweeney, H. L. \& Discher, D. E. Matrix Elasticity Directs Stem Cell Lineage Specification. Cell 126, 677-689 (2006).

22. Solon, J., Levental, I., Sengupta, K., Georges, P. C. \& Janmey, P. A. Fibroblast Adaptation and Stiffness Matching to Soft Elastic Substrates. Biophys. J. 93, 4453-4461 (2007).

23. Miner, J. H. Glomerular basement membrane composition and the filtration barrier. Pediatr. Nephrol. Berl. Ger. 26, 1413-1417 (2011).

24. Pavenstädt, H., Kriz, W. \& Kretzler, M. Cell Biology of the Glomerular Podocyte. Physiol. Rev. 83, 253-307 (2003).

25. Möller, C. C., Flesche, J. \& Reiser, J. Sensitizing the Slit Diaphragm with TRPC6 Ion Channels. J. Am. Soc. Nephrol. 20, 950-953 (2009). 
26. Vining, K. H. \& Mooney, D. J. Mechanical forces direct stem cell behaviour in development and regeneration. Nat. Rev. Mol. Cell Biol. 18, 728-742 (2017).

27. Ueber die Berührung fester elastischer Körper. J. Für Reine Angew. Math. Crelles J. 1882, 156-171 (2009).

28. Sneddon, I. N. The relation between load and penetration in the axisymmetric boussinesq problem for a punch of arbitrary profile. Int. J. Eng. Sci. 3, 47-57 (1965).

29. Vinckier, A. \& Semenza, G. Measuring elasticity of biological materials by atomic force microscopy. FEBS Lett. 430, 12-16 (1998).

30. Butt, H.-J., Cappella, B. \& Kappl, M. Force measurements with the atomic force microscope: Technique, interpretation and applications. Surf. Sci. Rep. 59, 1-152 (2005).

31. Martin, M. et al. Morphology and Nanomechanics of Sensory Neurons Growth Cones following Peripheral Nerve Injury. PLOS ONE 8, e56286 (2013).

32. Radmacher, M., Fritz, M., Kacher, C. M., Cleveland, J. P. \& Hansma, P. K. Measuring the viscoelastic properties of human platelets with the atomic force microscope. Biophys. J. 70, 556-567 (1996).

33. Boudou, T., Ohayon, J., Picart, C. \& Tracqui, P. An extended relationship for the characterization of Young's modulus and Poisson's ratio of tunable polyacrylamide gels. Biorheology 43, 721-728 (2006).

34. Saleem, M. A. et al. A conditionally immortalized human podocyte cell line demonstrating nephrin and podocin expression. J. Am. Soc. Nephrol. JASN 13, 630-638 (2002).

35. Bhadani Reena, Mitra Uttam Kumar. Studies on Water Absorbency of Polyacrylamide Hydrogels. J. Mater. Sci. Eng. B 5, 399-405 (2015). 
36. Grattoni, C. A., Al-Sharji, H. H., Yang, C., Muggeridge, A. H. \& Zimmerman, R. W. Rheology and Permeability of Crosslinked Polyacrylamide Gel. J. Colloid Interface Sci. 240, 601-607 (2001).

37. Okay, O. General Properties of Hydrogels. in Hydrogel Sensors and Actuators: Engineering and Technology (eds. Gerlach, G. \& Arndt, K.-F.) 1-14 (Springer Berlin Heidelberg, 2010).

38. Hale, L. J. et al. 3D organoid-derived human glomeruli for personalised podocyte disease modelling and drug screening. Nat. Commun. 9, 5167 (2018).

39. Walcott, S. \& Sun, S. X. A mechanical model of actin stress fiber formation and substrate elasticity sensing in adherent cells. Proc. Natl. Acad. Sci. 107, 7757-7762 (2010).

40. Reiser, J. \& Altintas, M. M. Podocytes. F1000Research 5, 114 (2016).

41. Wagner, N., Wagner, K.-D., Xing, Y., Scholz, H. \& Schedl, A. The Major Podocyte Protein Nephrin Is Transcriptionally Activated by the Wilms' Tumor Suppressor WT1. J. Am. Soc. Nephrol. 15, 3044-3051 (2004).

42. Palmer, R. E. et al. WT1 regulates the expression of the major glomerular podocyte membrane protein Podocalyxin. Curr. Biol. 11, 1805-1809 (2001).

43. Thomas, G., Burnham, N. A., Camesano, T. A. \& Wen, Q. Measuring the Mechanical Properties of Living Cells Using Atomic Force Microscopy. J. Vis. Exp. JoVE. 76, 50497 (2013). 